\section{rev Psi}

Revista de Psicología (UNLP)

https://revistas.unlp.edu.ar/revpsi

\title{
Intervenciones analíticas en la clínica actual. La dimensión de lo preliminar
}

\author{
Clarisa Moya $^{1}$ \\ Correspondencia \\ clarisamoya@gmail.com \\ Filiaciones institucionales \\ ${ }^{1}$ H.Z.G.A “Dr. Mario V. Larraín” de Berisso \\ (Argentina)
}

\section{Resumen}

En la actualidad, los practicantes del psicoanálisis que trabajan en instituciones de salud pública se confrontan con subjetividades marcadas por las características de la época, entre las cuales se encuentra la fragilidad del lazo social, el empuje a gozar y el pedido de soluciones rápidas al malestar. Esto se traduce clínicamente en nuevas formas de la demanda y presentaciones del padecimiento subjetivo atravesadas por el descreimiento en la palabra y la abolición del sujeto del deseo. Partiendo de la premisa "no hay contraindicaciones a priori al psicoanálisis", los analistas se enfrentan al desafío de otorgar una respuesta a lo que hay y a lo que no hay, mediante la invención de otros modos de intervenir orientados en una clínica de lo real.

\section{Palabras clave}

psicoanálisis | subjetividad | contemporaneidad | condiciones preliminares

\section{Cómo citar}

Moya, C. (2018). Intervenciones analíticas en la clínica actual. La dimensión de lo preliminar. Revista de Psicología, 17(2), 1420. doi: $10.24215 / 2422572 \mathrm{Xe} 018$
Recibido

13 jul. 2018

Aceptado

4 oct. 2018

Publicado

28 dic. 2018

Editor

Nicolás Alessandroni | Facultad de Psicología, Universidad Autónoma de Madrid (España)
ISSN

2422-572X

Licencia

(c) Copyright: Moya, C. Este trabajo se distribuye bajo una licencia de Cultura Libre CC-BY 4.0

Entidad editora

RevPsi es una publicación de la

Facultad de Psicología (Universidad

Nacional de La Plata, Argentina)

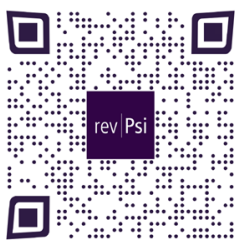

ACCESO ABIERTO DIAMANTE 


\section{Intervenções analíticas na clínica atual. A dimensão do preliminar}

\section{Resumo}

Atualmente, os profissionais da psicanálise que trabalham em instituições públicas de saúde são confrontados com subjetividades marcadas pelas características da época, dentre as quais a fragilidade do vínculo social, o impulso para desfrutar e a solicitação de soluções rápidas para o desconforto . Isso se traduz clinicamente em novas formas de demanda e apresentações do sofrimento subjetivo atravessado pela descrença na palavra e a abolição do sujeito do desejo. Partindo da premissa de que "não há contraindicações a priori à psicanálise", os analistas enfrentam o desafio de dar uma resposta ao que existe e ao que não existe, através da invenção de outros modos de intervenção orientados em uma clínica da realidade.

\section{Palavras-chave}

psicanálise | subjetividade | contemporaneidade | condições preliminares

\section{Analytical interventions in the current clinic. The dimension of the preliminary}

\section{Abstract}

At present, psychoanalysis practitioners working in public health institutions are confronted with subjectivities marked by the characteristics of our time, among which is the fragility of the social bond, the drive to pleasure and the demand for quick solutions to the discomfort. This translates clinically into new forms of the demand and presentations of the subjective suffering biased by the disbelief in the word and the abolition of the subject of desire. Starting from the premise "there are no a priori contraindications to psychoanalysis", analysts are faced with the challenge of providing a response to what there is and what there is not, through the invention of other modes of intervention oriented in a clinic of the real.

\section{Keywords}

psychoanalysis | subjectivity | contemporaneity | preliminary conditions 


\section{Aspectos destacados del trabajo}

- En la época actual predomina la búsqueda de soluciones rápidas y el rechazo al saber.

- El psicoanalista ofrece la escucha del malestar subjetivo, aun cuando ésta no fuese demandada.

- La intervención pretende conmover la rigidez pulsional o fantasmática que obtura la posibilidad de interrogación.

- Cuando se habilita la apuesta por la palabra, se constatan efectos terapéuticos sobre el malestar.

Las características de la época actual y los efectos que la contemporaneidad produce sobre la subjetividad, interpelan a los psicoanalistas que trabajan en instituciones de salud pública, invitándolos a pensar cómo sostener la vigencia de la experiencia analítica en la actualidad, y cómo responder frente a las nuevas formas de la demanda y presentaciones del padecimiento subjetivo.

Nos encontramos con una subjetividad marcada por la inconsistencia del Otro, la incitación al consumo y a la felicidad, la producción acelerada del objeto a en tanto tapón, la fragilidad de los lazos, el goce que afecta de modo exponencial al cuerpo, el empuje a ver y mostrar propiciado por la tecnología, el fracaso de los modos tradicionales de la regulación del goce, y el desarrollo del discurso de la ciencia. Estos elementos tienen como consecuencia la abolición del sujeto de la palabra y del deseo, y el rechazo al saber inconsciente y a la castración. Por lo tanto, las marcas de la época que lleva la subjetividad dan lugar a "posiciones subjetivas que parecen refractarias al análisis, que eluden confrontarse con las dificultades que exige la puesta en acto del deseo" (Rubistein, 2005, p. 242).

En el presente trabajo, se abordará el desafío para el practicante del psicoanálisis de pensar nuevos modos de intervenir, distintos a los que se desprenden de la clínica clásica, y de generar condiciones preliminares a las entrevistas preliminares. Esto es, lo que se podría llamar "lo preliminar de lo preliminar". En primera instancia, puesta en forma de la demanda, más que del síntoma, en una época marcada por la urgencia y la exigencia de soluciones mágicas y rápidas al malestar subjetivo.

\section{Indicaciones y ¿contraindicaciones? al psicoanálisis}

Freud ha planteado que en determinados casos el psicoanálisis es inaplicable, y en muchos otros, conviene no indicarlo. En Sobre psicoterapia (Freud, 1904/2008) plantea que el psicoanálisis está contraindicado para personas que consultan por orden de sus allegados, en aquellas situaciones donde sea preciso eliminar con 
rapidez fenómenos peligrosos (por ejemplo, una anorexia histérica), y en casos de toxicomanías y psicosis, entre otros. Sin embargo, dirá: "No descarto totalmente que una modificación apropiada del procedimiento nos permita superar esa contraindicación y abordar así una psicoterapia de las psicosis" (Freud, 1904/2008, p. 253). Tal perspectiva optimista será retomada en el artículo Nuevos caminos para la terapia psicoanalítica (Freud, 1918/2008), donde comenzará a delinear los desafíos que deberá enfrentar el psicoanálisis, y los nuevos desarrollos que se esperan para esta clínica.

Los post freudianos optaron por quedarse con el escepticismo del primer Freud, y durante mucho tiempo se detuvieron ante los límites de la eficacia del psicoanálisis. Es Lacan (1964/2006) quien retomará la posición ética de Freud en relación a no hacer de los consejos que otorgó una regla obligatoria. Partirá de la premisa "no hay contraindicaciones a priori al psicoanálisis", y nos moverá a ocuparnos de modos de sufrir ante los cuales se consideraba que nuestra clínica no tenía nada para hacer:

"El encuentro con un psicoanalista, en su conjunto, hace bien, ya que el objeto psicoanalista es asombrosamente versátil (...) Si el psicoanalista sabe ser objeto, no querer nada a priori por el bien del otro, no tener prejuicios en cuanto al buen uso que se puede hacer de él, ve el registro de las contraindicaciones reducirse, hasta el punto en que la contraindicación se decide, entonces, caso por caso" (Miller, 1999, p. 10).

Lo que nos autoriza éticamente a intervenir en tanto que analistas es lo que Lacan (1964/2006) denomina penar de más: "trop de mal: demasiado esfuerzo, demasiado sufrimiento, mal de sobra” (Lacan, 1964/2006, p. 174). Lo paradójico es que muchas veces el sujeto se aferra a su síntoma, a su modo de gozar, en tanto allí ha encontrado una solución para suplir la relación sexual que no existe. Por lo tanto, todo inicio de un tratamiento analítico tiene como punto de partida el consentimiento del sujeto, su apuesta a creer en la palabra y a encontrar otras modalidades de goce menos costosas. Para ello, algo de la demanda tiene que armarse y sostenerse.

En una entrevista en la Universidad de Yale, Lacan (1975) plantea, en relación a quienes consultan, que "se trata de hacerlos entrar por la puerta, que el análisis sea un umbral, que haya para ellos una verdadera demanda" (párr. 4), definida por el autor como la demanda de querer desembarazarse de un síntoma. En esa demanda, aclara, "es necesario que algo empuje" (párr. 9). En la medida en que esto no siempre está de entrada, las maniobras analíticas apuntarán a que el paciente pueda articular el lugar de una falta, de una hiancia, donde sea posible localizar un punto de demanda (Rubistein, 2014), en el que se sitúe aquello que empuja en lo pulsional.

Ahora bien, ¿con qué subjetividades nos encontramos en la clínica actual? 


\section{La clínica de la urgencia}

Según Miller, "en los tiempos que corren, uno ya no tiene tiempo de tomarse el tiempo" (Miller, 2015, p. 25). La urgencia, la prisa, la aceleración y la inmediatez trascienden las paredes de una guardia e invaden cada espacio de los dispositivos hospitalarios y de salud pública. Los tiempos que corren no es sólo el tiempo que pasa, es el movimiento acelerado de la civilización, lo que a su vez delimita un modo de estar en la actual sociedad, un modo de gozar en ella, dirá Miller.

Nos encontramos con sujetos que no suponen ni demandan un saber sobre su síntoma, el cual se muestra reacio a la articulación significante. No pueden decir demasiado sobre su sufrimiento y, mucho menos, sobre las hipótesis en relación a la causa. El pedido es el de una solución rápida y efectiva al malestar, sin demasiados costos ni trabajo para el consultante.

Sujetos mudos, que revelan el mutismo del goce. O sujetos que hablan mucho, pero dicen poco: goce del blábláblá que reactualiza el "déjeme hablar" de Emmy von N. y la "talking cure" de Anna O. Pura catarsis preanunciada por los tan frecuentes "no tengo con quién hablar", "necesito descargar", como respuestas a la pregunta sobre lo que el paciente espera de un tratamiento psicológico. Goce autista del pârletre, en la época del contar todo, y siempre decir lo mismo. "Hay así, entonces, un goce de decir, independientemente del contenido semántico que vehiculiza. Es a la vez goce de hacerse escuchar, goce del mero contacto con el Otro" (Blanchet, 2015, p. 237).

Siguiendo a Rivas (2007), se observan ciertas presentaciones del malestar contemporáneo bajo la forma de fenómenos del desorden pulsional (como las toxicomanías y los trastornos de la conducta alimentaria), de deslocalización del goce, y diversas patologías del acto, (como pasajes al acto y acting out) donde la palabra es reemplazada por la acción. En las instituciones hospitalarias son frecuentes las interconsultas por "intentos de autoeliminación”, en las que el paciente ha ingerido pastillas o bien se ha autoinfligido cortes. Será tarea del analista elucidar si efectivamente esto responde a un intento de suicidio o bien se trata de un llamado al Otro.

Miller (2015) hablará de psicoanálisis líquido, tomando como referencia el adjetivo con el cual Z. Bauman caracterizó a la civilización actual, donde vemos una aceleración en los cambios, una difusión de las fronteras y una fluidez y volatilización en las relaciones humanas. Un efecto de desestructuración que impacta también a nivel de la práctica analítica, en tanto ya no nos encontramos con las histéricas de antaño sino con presentaciones en las que las herramientas que nos legó Freud y el primer Lacan son insuficientes.

La clínica de hoy ya no responde a la solidez de la época del psicoanálisis de la estructura. En este marco, la propuesta lacaniana de psicoanálisis nodal se ajusta al efecto de mutación que puede llegar a tener la acción de una fuerza mínima: tirar cuerdas. Tironeo que repercutirá en los posibles anudamientos, en aquello que engancha y sostiene a un sujeto, entendiendo al nudo como "una articulación entre 
lo líquido y lo que subsiste de estructura" (Miller, 2015, p. 210).

La subjetividad de la época obliga del lado del practicante del psicoanálisis a pensar otro tipo de intervenciones, que no se dirigen directamente al entramado significante, al sentido, aunque siempre tienen como brújula generar un efecto de división subjetiva e introducir en el discurso de sujeto algo de la dimensión simbólica. Se trata de un psicoanálisis que se compromete con lo real, al tiempo que ya no descarta la posibilidad de intervenciones en lo imaginario, siendo que el último Lacan postula la equivalencia de los tres registros, y en este punto, una intervención en uno de ellos repercutirá en los otros, dado el entrelazamiento real-simbólico-imaginario.

Al desubjetivar su padecimiento y localizar la causa del mismo en el campo del Otro, el sujeto intenta "no querer saber nada sobre la falta". En esta línea, Recalcati (2004) señala la impotencia actual que caracteriza el presupuesto de la clínica clásica basado en la transformación de la voluntad de curar en la voluntad de saber, lo que propiciaría una exigencia de desciframiento.

$\mathrm{Al}$ respecto, Miller se preguntará:

\begin{abstract}
“Hay que mantener el concepto de saber, sin el cual no se sostienen las nociones de ciframiento y de desciframiento? Me parece que si la estructura adecuada al psicoanálisis líquido es el nudo, como lo indicaba Lacan, entonces hay que relativizar, o incluso desechar, el desciframiento y preferir el corte del redondel de cuerda, ya que, si el psicoanálisis nodal de Lacan pone en escena la acción de tirar para mostrar sus aspectos, implica también otra acción, una acción quirúrgica: cortar” (Miller, 2015, p. 216-217).
\end{abstract}

Allí donde ya no predominan las formaciones del inconscientes, pasibles de descifradas, tenemos los acontecimientos de goce, mudez pulsional. En la suspensión de la sesión, que implica un corte en el discurso del sujeto, se busca generar la emergencia de la pregunta por el deseo del analista, instalar un enigma a descifrar: “Qué quieres?”. Se espera que el sujeto se vea movido a la producción de significantes por fuera de la sesión, partiendo de la insistencia de lo último que dijo, momento de interrupción. Similar orientación tiene la interpretación que apunta al fuera del sentido, buscando detener la apertura significante infinita y generar una operación de reducción que toque lo inerte del síntoma y la repetición del goce.

Esencialmente, se pretende introducir una pausa, una detención, momento de comprender, lo cual a su vez requiere un tiempo, el de la espera de efectos que a posteriori verifiquen que algo de ese goce se conmovió, precipitando el momento de concluir.

\title{
La oferta del psicoanálisis
}

La clínica de hoy nos enseña que en muchos casos, puede no haber discurso analítico, pero si hay un practicante del psicoanálisis, habrá puesta en juego del deseo del 
analista, aun cuando el goce no parece molestar al paciente. En el Seminario 17, Lacan afirma que "es preciso, lo repito, no proceder por medio del psicoanálisis, sino tal vez ser un psicoanalista, si es que eso existe" (Lacan, 1970/2006, p. 96), incluso en aquellos casos en los que no estemos tan seguros de lo que el psicoanálisis puede ofrecer. Se trata de trata de aprovechar la oportunidad que ha tenido ese sujeto de encontrarse con un practicante del psicoanálisis, y que ese encuentro busque operativizar la frase lacaniana: "con oferta, he creado demanda" (Lacan, 1958/1987, p. 588). Es el acto ético de que los dichos de un sujeto puedan ser escuchados para localizarlo en su decir.

Por supuesto que quedará del lado del paciente la decisión de arriesgarse a la aventura subjetiva que implica una experiencia analítica. $\mathrm{O}$ bien elige quedarse capturado por la mudez de su cuerpo y del goce, o bien se juega a dejarse llevar por la palabra, la cual no le da ninguna garantía respecto del sitio donde lo conducirá (Chiriaco, 2012). En este último caso, se habilitan condiciones para transformar la oferta de la escucha de un analista, en una demanda al analista.

\section{Referencias}

Baudini, S. (2015). Scilicet - El cuerpo hablante: sobre el inconsciente en el siglo XXI. Buenos Aires: Grama.

Brodsky, G. (2014). La clínica y lo real. Buenos Aires: Grama.

Chiriaco, S. (2012). L’ultime menace [La última amenaza]. En Le désir foudroyé. Sortir $d u$ traumatisme par la psychanalyse (pp. 155-169). Paris: Navarin [L. Volta, Trad.].

Freud, S. (1918/2008). Nuevos caminos para la terapia psicoanalítica. En Obras completas. Tomo XVII (pp. 151-164). Buenos Aires: Amorrortu.

Freud, S. (1904/2008). Sobre psicoterapia. En Obras completas. Tomo VII (pp. 243-272). Buenos Aires: Amorrortu.

Lacan, J. (1975). Entrevista a Jacques Lacan en la Universidad de Yale. (O. M. Máter y A. Freschi, Trans.). Recuperado a partir de нттр://Www. ELSIGMA.COM/ENTREVISTAS/ENTREVISTAA-JACQUES-LACAN-EN-LA-UNIVERSIDAD-DEYALE/11644

Lacan, J. (1958/1987). La dirección de la cura y los principios de su poder. En Escritos 2 (pp. 559616). Buenos Aires: Siglo XXI.

Lacan, J. (1964/2006). El seminario. Libro 11. Los cuatro conceptos fundamentales del psicoanálisis. Buenos Aires: Paidós.

Lacan, J. (1970/2006). El seminario. Libro 17. El reverso del psicoanálisis. Buenos Aires: Paidós.
Miller, J.-A. (1999). Las contraindicaciones al tratamiento psicoanalítico. El Caldero de la Escuela. Revista de la EOL, 69, 7-12.

Miller, J.-A. (2015). Todo el mundo es loco. Buenos Aires: Paidós.

Muñoz, P. (2009). La invención lacaniana del pasaje al acto. De la psiquiatría al psicoanálisis. Buenos Aires: Manantial.

Recalcati, M. (2004). La cuestión preliminar en la época del Otro que no existe. Virtualia, Revista digital de la EOL, 10, 2-6.

Rivas, E. (2007). Pensar las psicosis. Buenos Aires: Grama.

Rubistein, A. (2005). Para una política del psicoanálisis en la época actual. En A. Glaze (Ed.), Una práctica de la época. El psicoanálisis en lo contemporáneo (pp. 241-259). Buenos Aires: Grama.

Rubistein, A. (2014). Consulta, admisión, derivación Buenos Aires: EUdeBA.

Sotelo, I. (2004). La guardia, la admisión, la primera consulta: una coyuntura de emergencia. En G. Belaga (Ed.), La urgencia generalizada. La práctica en el hospital (p. 97). Buenos Aires: Grama. 